\title{
Stratified natural convection flow in rectangular open cavities
}

\author{
M. Nateghi ${ }^{1} \quad$ J. Patterson ${ }^{2} \quad$ S. W. Armfield ${ }^{3}$
}

(Received 27 November 2006; revised 7 November 2007)

\begin{abstract}
We explore natural convection in a two dimensional rectangular open cavity with thermally stratified ambient for both transient and steady-state flow. The left hand vertical wall of the cavity is heated and the facing right hand wall is open. The top and bottom are insulated. Numerical solutions are obtained for Rayleigh numbers varying from $10^{5}$ to $10^{10}$ with Prandtl number 1.0. This article focuses on the effect of stratification of the ambient fluid on the unsteady behaviour of the natural convection flow in rectangular open cavities. Results show that the flow is steady at low Rayleigh number and becomes unsteady at sufficiently high Rayleigh number $\left(5.4 \times 10^{8}\right)$. Streamline and temperature contour plots give a better understanding of the heat transfer and the flow mechanisms inside the cavity.
\end{abstract}

See http://anziamj.austms.org.au/ojs/index.php/ANZIAMJ/article/view/191 for this article, (c) Austral. Mathematical Soc. 2007. Published November 25, 2007. ISSN 1446-8735 


\section{Contents}

1 Introduction

C543

2 Numerical method

C544

3 Results and discussion

C546

3.1 Low Rayleigh number and steady state flow . . . . . . . . C548

3.2 High Rayleigh number and unsteady flow . . . . . . . . . C548

4 Conclusions

C553

References

C555

\section{Introduction}

Two-dimensional natural convection flow in enclosures has received considerable attention in the past $[1,3,6,10,11]$ whereas the related problem of partial enclosures and open cavities, has received very little attention $[5,7,9]$. One of the reasons for this can be traced to the difficulty in specifying the proper boundary conditions for an open cavity configuration. Javam and Armfield [8] showed that at Prandtl number $\operatorname{Pr}=0.7$ in the open cavity with background stratification the flow undergoes bifurcations of the same type as observed for the closed cavity with, for a sufficiently high Rayleigh number low and high frequency signals observed.

In this study, unsteady natural convection flow in two dimensional open cavities with thermally stratified ambient has been investigated. The cavity has one heated vertical wall facing a vertical opening, with top and bottom insulated. Results were obtained for Rayleigh number varying from $10^{5}$ to $10^{10}$ with Prandtl number $\operatorname{Pr}=1.0$. The purpose is to determine the basic structure of the flow and to obtain the critical Rayleigh number asso- 
ciated with bifurcation and transition to the chaotic flow. Streamline and temperature contour plots illustrate the dependence upon Rayleigh number of the basic flow patterns.

\section{Numerical method}

The equations considered govern the motion of an incompressible, viscous and stable temperature stratified flow. We assume that the flow is twodimensional and unsteady with constant properties. Using the Boussinesq approximation, the continuity, momentum, and energy equations governing two-dimensional stratified natural convection flow are written in nondimensional form as follows:

$$
\begin{aligned}
& \frac{\partial u}{\partial x}+\frac{\partial v}{\partial y}=0 \\
& \frac{\partial u}{\partial t}+\frac{\partial(u u)}{\partial x}+\frac{\partial(v u)}{\partial y}=-\frac{\partial p}{\partial x}+\left(\frac{\partial^{2} u}{\partial x^{2}}+\frac{\partial^{2} u}{\partial y^{2}}\right) \\
& \frac{\partial v}{\partial t}+\frac{\partial(u v)}{\partial x}+\frac{\partial(v v)}{\partial y}=-\frac{\partial p}{\partial y}+\left(\frac{\partial^{2} v}{\partial x^{2}}+\frac{\partial^{2} v}{\partial y^{2}}\right)+\frac{\operatorname{Ra}}{\operatorname{Pr}} T \\
& \frac{\partial T}{\partial t}+\frac{\partial(u T)}{\partial x}+\frac{\partial(v T)}{\partial y}=\frac{1}{\operatorname{Pr}}\left(\frac{\partial^{2} T}{\partial x^{2}}+\frac{\partial^{2} T}{\partial y^{2}}\right)-v \frac{d T_{b}}{d y}
\end{aligned}
$$

The Rayleigh number and Prandtl number are defined respectively as

$$
\mathrm{Ra}=\frac{g \beta \Delta T H^{3}}{\nu \kappa},
$$

and

$$
\operatorname{Pr}=\frac{\nu}{\kappa},
$$

where $g$ is the acceleration due to gravity, $\beta$ the coefficient of thermal expansion, $t$ is the time, $T$ and $T_{b}$ are the fluctuating and background temperature 


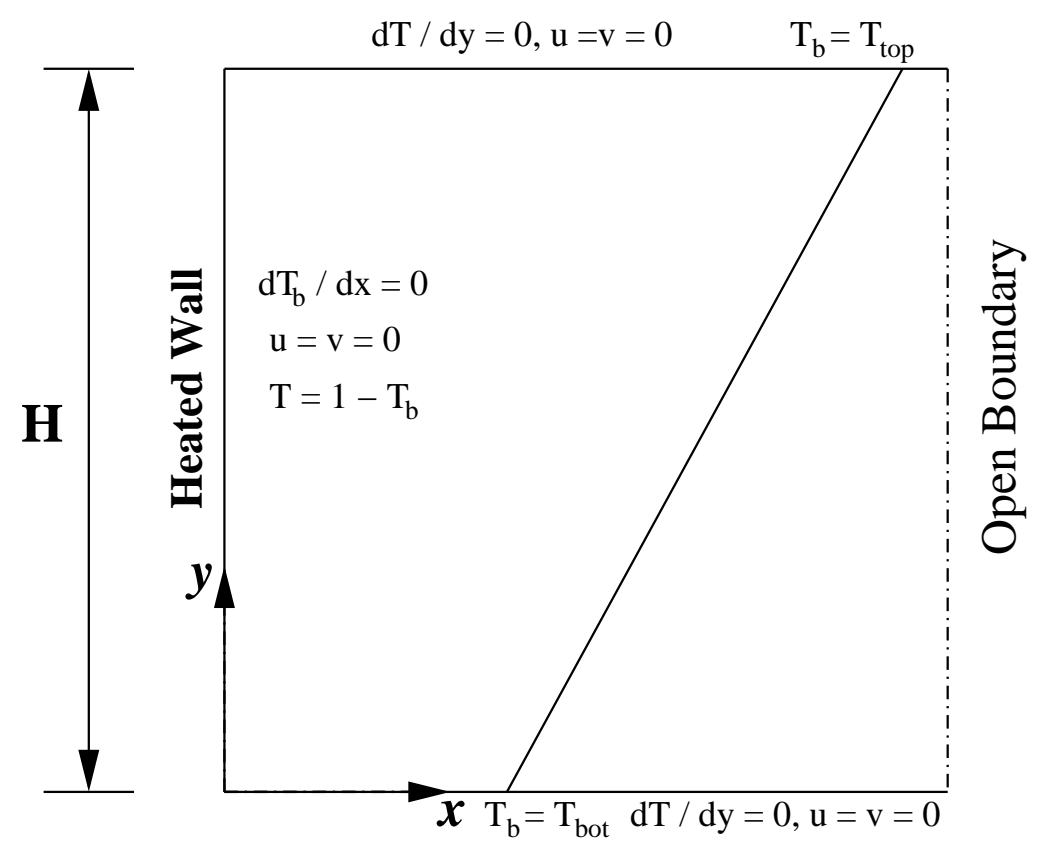

Figure 1: Geometry and boundary condition.

respectively, $\Delta T$ is the total temperature variation, $H$ is the height of the cavity and $\kappa$ the thermal diffusivity. $u$ is the velocity component in the horizontal $x$-direction and $v$ is the velocity component in the vertical $y$-direction. The geometry of the open cavity, the coordinate system and boundary conditions for the present study are shown in Figure 1. On the open boundary the $x$ derivatives of the velocities are set to zero, while for positive $u$-velocity $\partial T / \partial x=0$ and for negative $u$-velocity $T=0$. Initially the fluid is at rest and isothermal $(T=0)$ and at time $t=0$ the left side wall is instantaneously heated to a non-dimensional temperature of $T=1-T_{b}$.

The equations of motion were solved using a fractional step, NavierStokes solver. The scheme is defined on a non-staggered mesh in which all the variable are stored at the same grid locations, using finite volumes, with standard second order central differences used for the viscous terms, the pres- 
sure gradient and divergence terms. The advective terms are approximated using QUICK [8]. The momentum and temperature terms are inverted using a limited iteration ADI solver and Poisson pressure correction equation uses a preconditioned restarted GMRES solver [2]. The scheme is similar to that presented for a staggered mesh in Armfield and Street [4].

A non-uniform mesh is used allowing grid nodes to be concentrated in the region of the heated wall and the upper and lower boundaries. The origin is located at the bottom left corner of the domain with $y$ increasing up the hot wall and $x$ increasing horizontally into the domain. The horizontal mesh size adjacent to the hot wall is $\Delta w_{x}=0.001$ with a grid stretching factor of 1.07 per cell unit until $x=0.1$, the stretching factor is then gradually reduced until the grid becomes uniform. The vertical mesh size adjacent to the insulated boundaries is $\Delta w_{y}=0.001$ with the same stretching factor per cell unit until $y=0.1$ for the lower domain and $y=0.9$ for the upper domain. The grid stretching factor is then gradually reduced until the grid becomes uniform in the interior. This gives a basic grid of $129 \times 172$ nodes for aspect ratio 0.5 (Height/Width) which is used with a basic time step of $\Delta t=2.5 \times 10^{-7}$. Mesh and time step dependence tests have been carried out by comparing the solution obtained on the basic mesh of $129 \times 172$ and two more meshes of $87 \times 111$ and $245 \times 357$ nodes. The comparison between the three mesh sizes results show very small variations, indicating that the basic mesh and time step provide sufficient resolution.

\section{Results and discussion}

Results are presented for a wide range of Rayleigh numbers varying from $\mathrm{Ra}=10^{5}$ to $\mathrm{Ra}=10^{10}$ with $\mathrm{Pr}=1.0$ in rectangular open cavities. Initially the fluid in the cavity is stationary with a background temperature $T_{b}$ and the fluctuating temperature set to zero $T=0$. At time $t=0$ the left hand vertical side wall is instantaneously heated to a non-dimensional temperature 

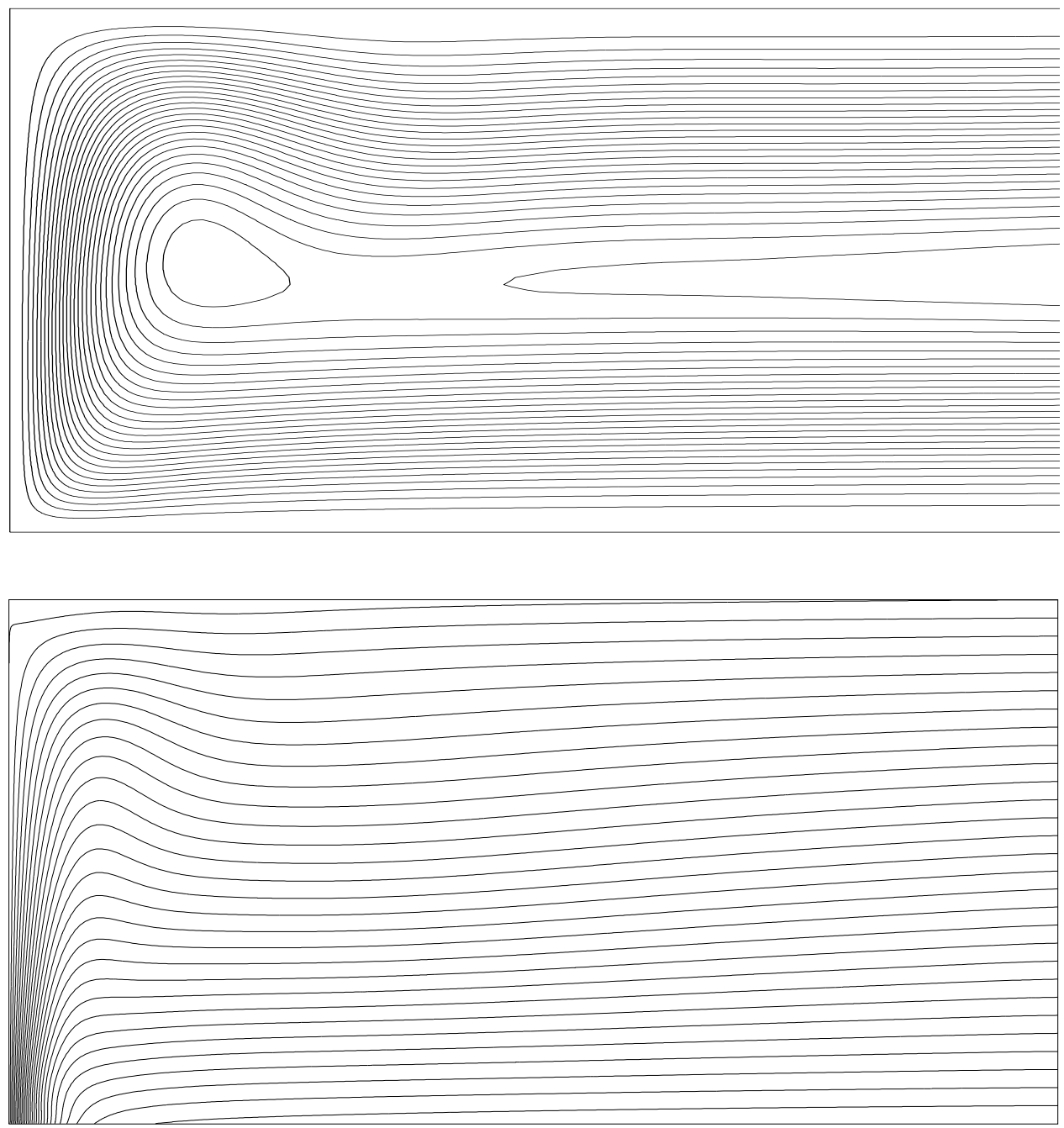

Figure 2: Streamline and temperature contours of stratified flow at $\mathrm{Ra}=$ $10^{5}$. 
of $T=1-T_{b}$ (the total temperature is $T+T_{b}$ ). The background temperature gradient, $d T_{b} / d y=2.0$ with $T_{b}(x, 0)=-1$, is constant for all simulations.

\subsection{Low Rayleigh number and steady state flow}

Figure 2 contains the fully developed stream-function (top) and temperature (bottom) contours for $\mathrm{Ra}=10^{5}$ with the hot wall on the left and the open boundary on the right at time $t=0.25$. The flow enters the cavity from the lower half of the open boundary and exits the cavity through the upper part of the open boundary, forming a hot intrusion that is driven across the cavity by a combination of momentum and buoyancy. The entrainment and de-entrainment regions are observed to be almost identical. As is seen in Figure 2 the isotherms show a thick thermal boundary layer is formed and travels up the heated wall entraining the ambient fluid over the lower half of its height and discharging over the upper half. The discharge of fluid from the upper part of the boundary layer occurs smoothly. The time series of the temperature (not shown in here) show that the flow is steady at this Rayleigh number as expected based on the critical Rayleigh number for the convective instability of a natural convection boundary layer as reported by Armfield and Janssen [3].

\subsection{High Rayleigh number and unsteady flow}

Figure 3 shows the fully developed stream-function (top) and temperature contours (bottom) for $\mathrm{Ra}=5.3 \times 10^{8}$ at $t=0.25$. The basic flow structure is the same as that observed in Figure 2 for the lower Rayleigh number flow, with a natural convection boundary layer adjacent to the heated wall entraining fluid from the lower part of the open cavity and open boundary, which is then discharged into an intrusion beneath the upper boundary. However, the discharge of fluid from the upper part of the boundary layer is quite different 

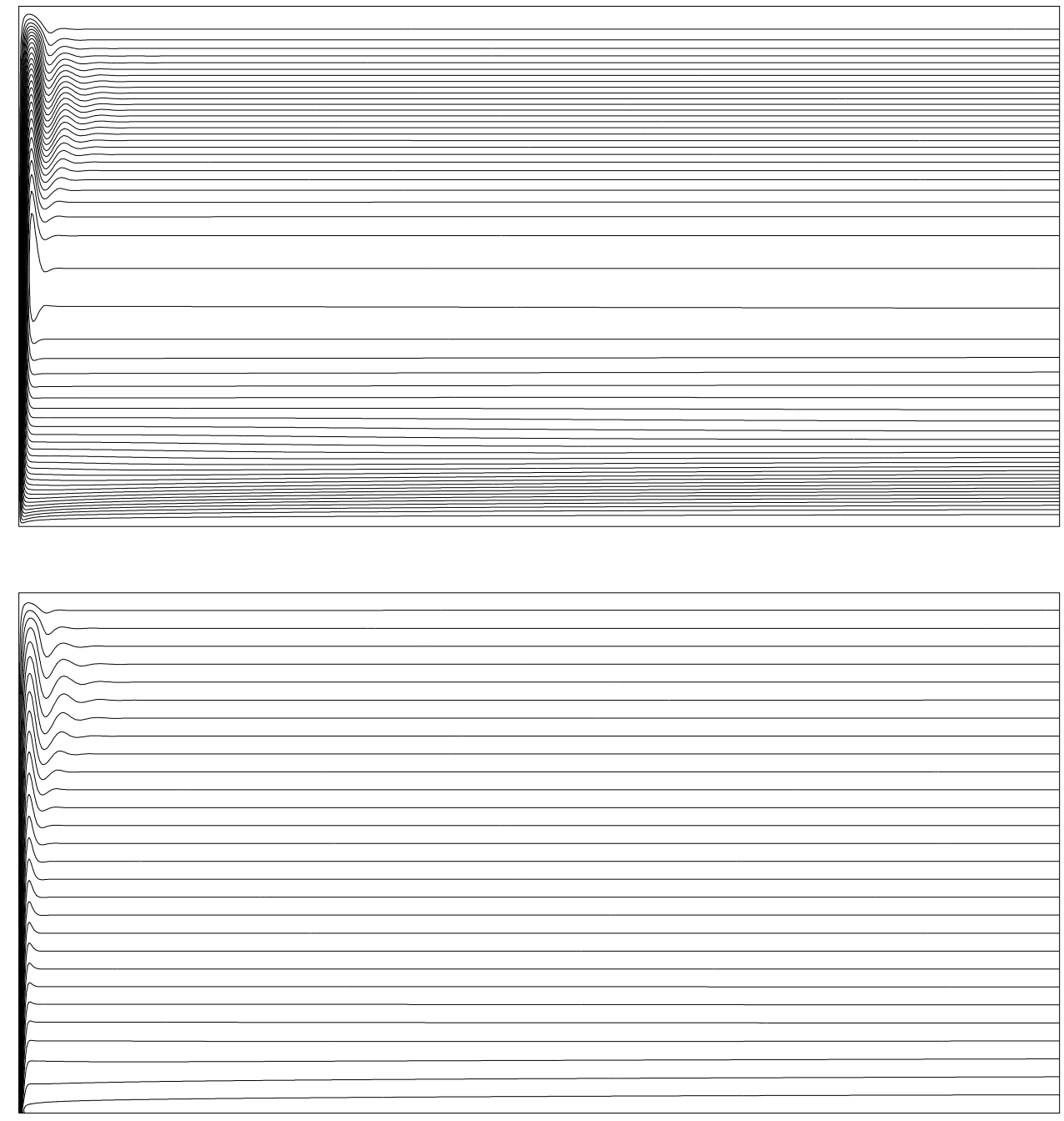

Figure 3: Streamline and temperature contours of stratified flow at Ra $=$ $5.3 \times 10^{8}$. 

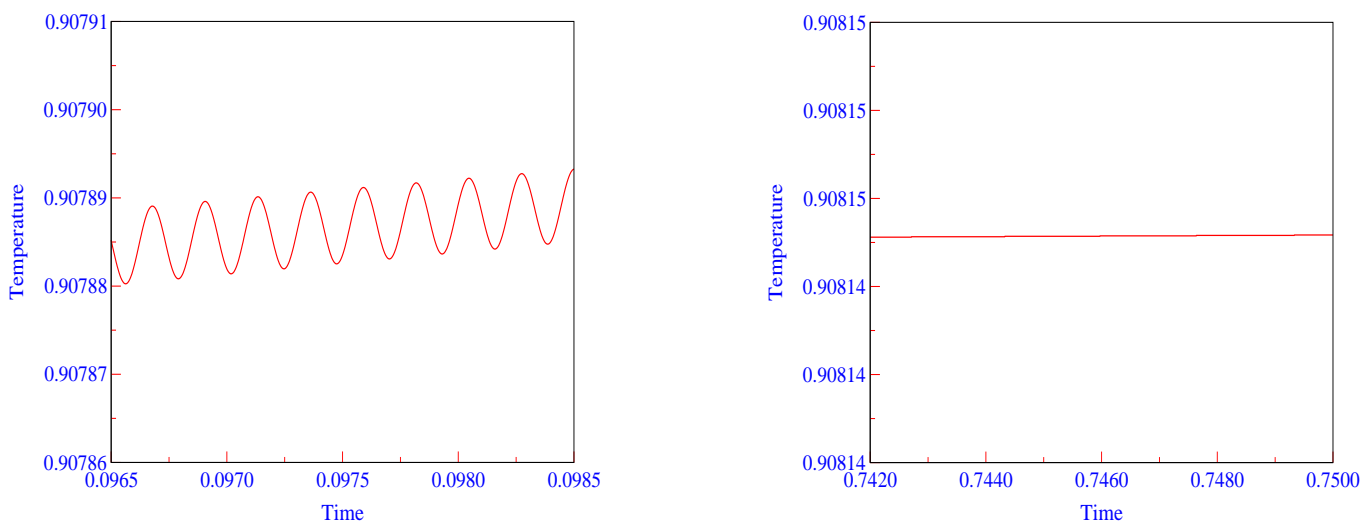

Figure 4: Temperature time series for $\mathrm{Ra}=5.3 \times 10^{8}$ at different times.
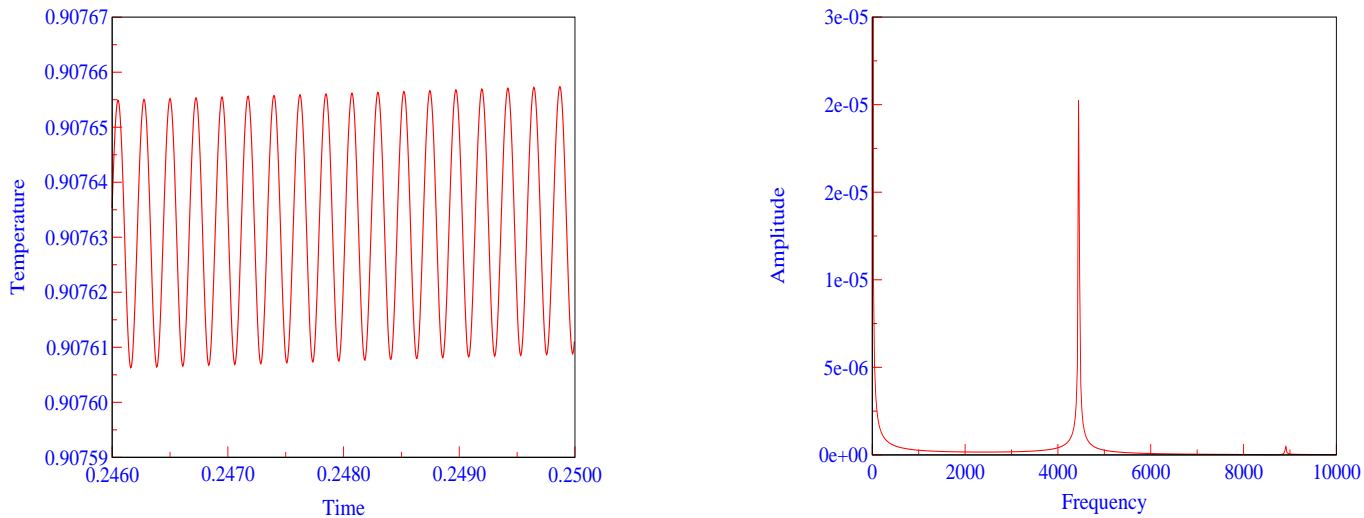

FiguRE 5: Temperature time series and the associated spectra for $\mathrm{Ra}=$ $5.4 \times 10^{8}$. 
from that of the lower Rayleigh number result shown above. The hot intrusion in the upstream region exhibits a wave like structure. The discharge passes smoothly into the intrusion through the wave structure, and exits the domain. The thermal boundary layer is thinner than that observed for the lower Rayleigh number, which is expected as the boundary layer thickness scales as $\delta_{T}=H / \mathrm{Ra}^{1 / 4}[11]$.

Figure 4 contains the temperature time series at a point adjacent to the hot wall $(x=0.004, y=0.916)$ for $\mathrm{Ra}=5.3 \times 10^{8}$. The time series show that the flow is unsteady with a quasi-periodic behavior at early time stage. However, the flow ultimately becomes steady at larger time. Therefore, this Rayleigh number is classified as a sub-critical flow. Figure 5 presents temperature time series adjacent to the hot wall and the associated spectra for $\mathrm{Ra}=5.4 \times 10^{8}$. The flow structure is similar to that observed for $\mathrm{Ra}=5.3 \times 10^{8}$ and for brevity temperature and stream-function contours are not shown. The temperature time series show the flow is unsteady and has a periodic behavior. The spectra of the time series demonstrate the signal has a dominant frequency, determined using Fourier transforms to be $f=4440$. Therefore the flow undergoes a bifurcation to unsteady flow at $\mathrm{Ra}=5.4 \times 10^{8}$. This Rayleigh number is defined as supercritical and the critical Rayleigh number then lies between $5.3 \times 10^{8}$ and $5.4 \times 10^{8}$.

Figure 6 shows the stream-function (top) and temperature contours (bottom) for the fully developed flow at $\mathrm{Ra}=2.0 \times 10^{9}$ at $t=0.25$. The overall flow structure at this Rayleigh number is similar to that observed for $\mathrm{Ra}=5.3 \times 10^{8}$. However, the discharge of fluid from the upper part of the boundary layer is different from that observed for the lower Rayleigh number, with vertical perturbations visible in this region. These perturbations are associated with waves traveling up the boundary layer and passing into the intrusion. These waves are also seen in Figure 7 which contains the temperature time series adjacent to the hot wall $(x=0.004, y=0.916)$ and the associated spectra for $\mathrm{Ra}=2.0 \times 10^{9}$. The temperature time series indicate the flow is unsteady and has a periodic behavior. The spectra of the time 

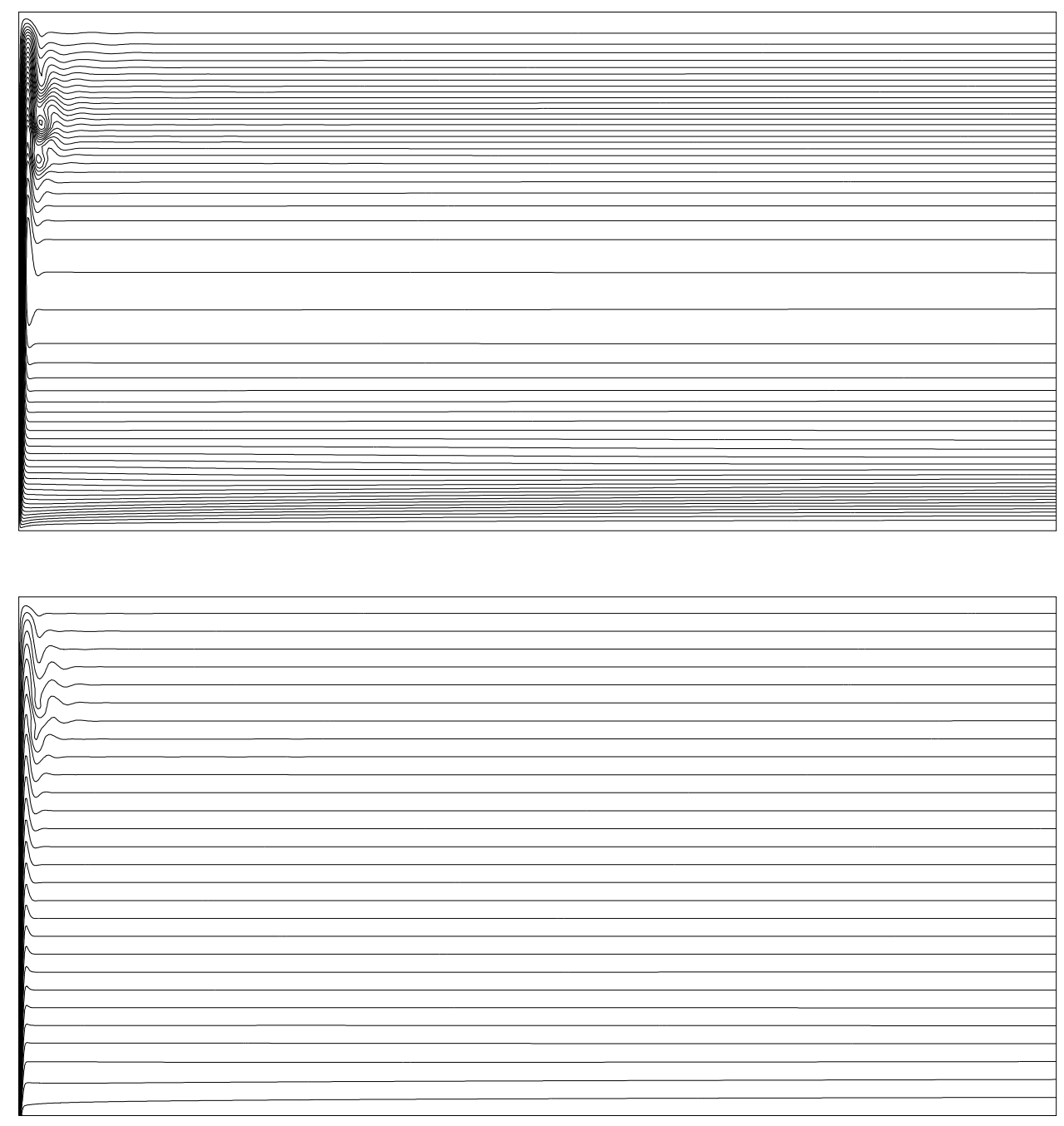

FiguRE 6: Streamline and temperature contours of stratified flow at Ra $=$ $2 \times 10^{9}$. 

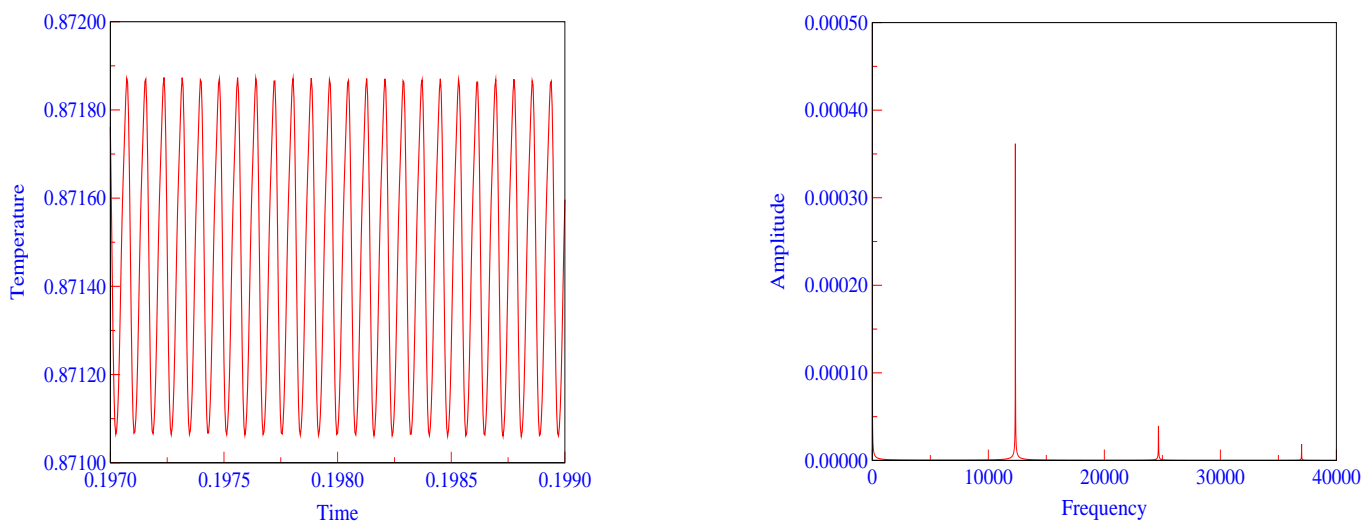

FiguRE 7: Temperature time series and the associated spectra for $\mathrm{Ra}=$ $2 \times 10^{9}$.

series show the signal has a dominant frequency of $f=12330$. Figure 8 presents the fully developed stream-function contours (top) and temperature time series adjacent to the hot wall with the associated spectra (bottom) for $\mathrm{Ra}=2.2 \times 10^{9}$. The flow structure is different to that observed for $\mathrm{Ra}=2.0 \times 10^{9}$ with perturbations observed throughout the cavity indicating a transition to a chaotic multi-modal flow has occurred. The spectra of the time series demonstrate the signal has an overall dominant frequency with a broad banded structure.

\section{Conclusions}

Natural convection in rectangular open cavities with stratified ambient, for Rayleigh numbers varying from $\mathrm{Ra}=10^{5}$ to $\mathrm{Ra}=10^{10}$ with Prandtl number $\operatorname{Pr}=1.0$, has been investigated. Flow enters the cavity from the lower half of the open boundary and leaves the cavity at the upper half of the open boundary. A boundary layer forms on the heated wall entraining fluid from the interior over the lower boundary layer region and discharging it into an 

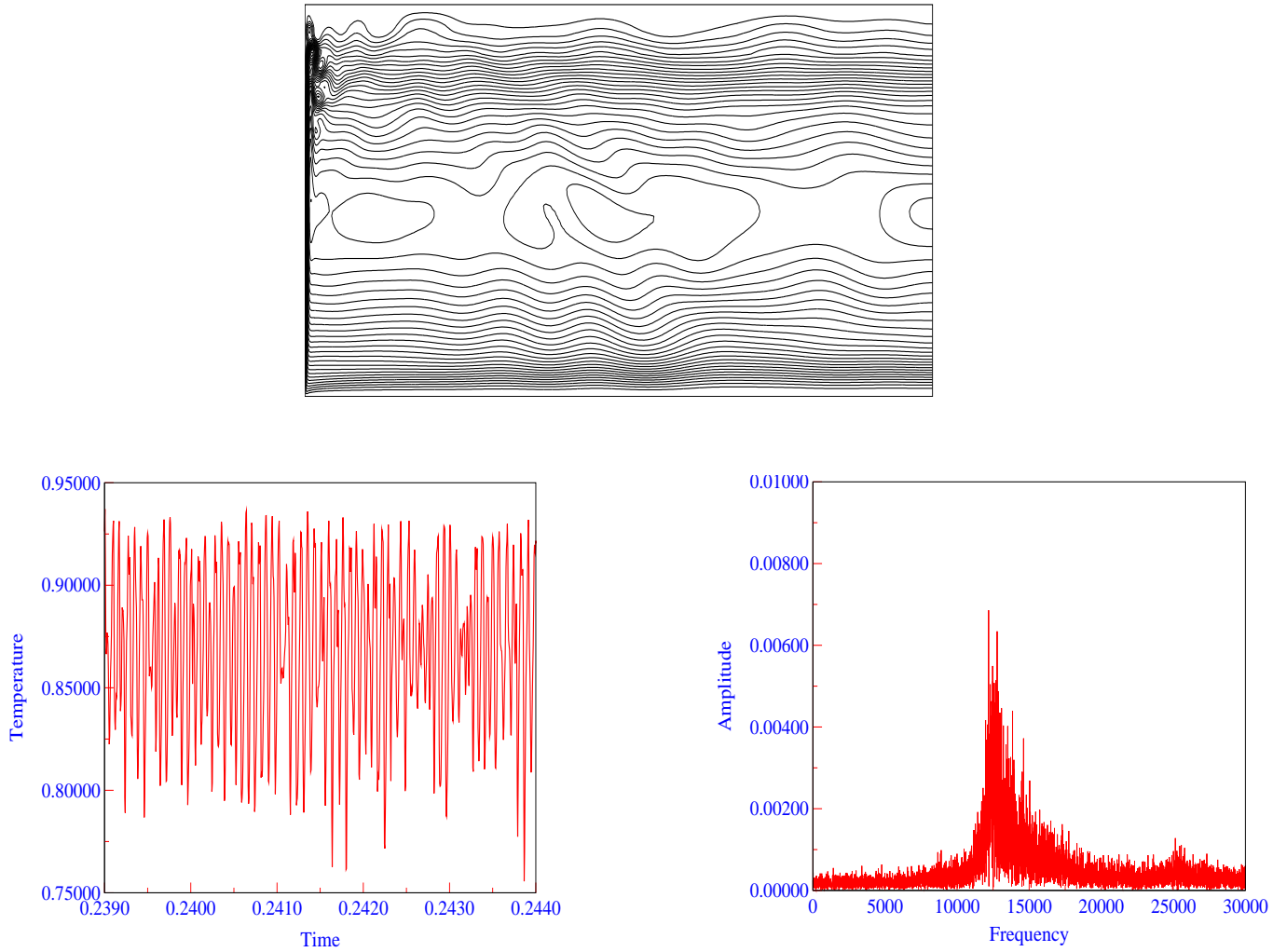

Figure 8: Streamline contours (top) and temperature time series with the associated spectra (bottom) for $\mathrm{Ra}=2.2 \times 10^{9}$. 
intrusion in the upper region. As the flow passes from the boundary layer to the intrusion it forms a wave-like structure. As the Rayleigh number is increased the boundary layer becomes thinner and the length of the wavelike structure reduces. The flow bifurcates to a periodic unsteady mode at $\mathrm{Ra}=5.4 \times 10^{8}$ and the critical Rayleigh number for this bifurcation was found to be between $5.3 \times 10^{8}$ and $5.4 \times 10^{8}$. A similar bifurcated periodic flow is observed at $\mathrm{Ra}=2 \times 10^{9}$; however, the amplitude is now considerably increased with the waves clearly visible in the steam-lines in the upper part of the boundary layer. Flow animations have shown these are traveling waves, amplifying in their direction of travel, and are therefore most likely to be associated with the known convective instability of the natural convection boundary layer [3]. A further increase in the Rayleigh number, to $\mathrm{Ra}=2.2 \times 10^{9}$, leads to a general broad-banded spectra and the flow undergoes a further transition between $\mathrm{Ra}=2 \times 10^{9}$ and $\mathrm{Ra}=2.2 \times 10^{9}$ to a multi-modal chaotic structure.

Acknowledgments The authors gratefully acknowledge financial support from the Australian Research Council.

\section{References}

[1] S. W. Armfield and J. C. Patterson. Direct simulation of wave interactions in unsteady natural convection in a cavity. Intl. J. Heat Mass Transfer., 34:929-940, 1991. C543

[2] S. W. Armfield. Ellipticity, accuracy, and convergence of the discrete Navier-Stokes equations. J. Comput. Phys., 114:176-184, 1994. C546

[3] S. W. Armfield and R. Janssen. A direct boundary-layer stability analysis of steady-state cavity convection flow. Int. J. Heat Fluid Flow., 17:539 (1996). C543, C548, C555 
[4] S. W. Armfield and R. Street. The fractional-step method for the Navier-Stokes equations on staggered grids: the accuracy of three variations. J. Comput. Phys., 153:660-665, 1999. C546

[5] Y. L. Chan and C. L. Tien. A numerical study of two-dimensional laminar natural convection in shallow open cavities. Int. J. Heat and mass transfer., 28:603-612, 1985. C543

[6] J. A. Janssen and R. A. W. M. Henkes. Influence of Prandtl number on instability mechanisms and transition in a differentially heated square cavity. Int. J. Fluid Mech., 290:319-344, 1995. C543

[7] A. Javam and S. W. Armfield. Stability and transition of stratified natural convection flow in open cavities. J. Fluid Mech., 445:285-303, 2001. C543

[8] B. P. Leonard. A stable and accurate convective modeling procedure based on quadratic upstream interpolation. Comput. Meth. Appl. Mech. Engng., 19:59-98, 1979. C543, C546

[9] M. Nateghi and S. W. Armfield. Natural convection flow of air in an inclined open cavity. ANZIAM J., 45:870-890, 2004. C543

[10] J. C. Patterson and J. Imberger. Unsteady natural convection in a rectangular cavity. Int. J. Fluid Mech., 100:65-86, 1980. C543

[11] J. C. Patterson and S. W. Armfield. Transient features of natural convection in a cavity. J. Fluid Mech., 219:469-498, 1990. C543, C551 


\section{Author addresses}

1. M. Nateghi, School of Aerospace, Mechanical and Mechatronic Engineering, Sydney University, Sydney, Australia. mailto:mnateghi@tpg.com.au

2. J. Patterson, James Cook University, Australia. mailto: john.patterson@jcu.edu.au

3. S. W. Armfield, School of Aerospace, Mechanical and Mechatronic Engineering, Sydney University, Sydney, Australia. mailto:armfield@aeromech.usyd.edu.au 